Journal of Animal and Veterinary Advances $11(8): 1183-1187,2012$

ISSN: $1680-5593$

(C) Medwell Journals, 2012

\title{
Molecullar Typing, Prevalence of netB and Antimicrobial Susceptibility among Clinical Isolates of Clostridium perfringens from Chickens in Southern China
}

\author{
Caiyan Wu, Shenquan Liao, Nanshan Qi, Xinyu Peng, Minna Lv and Mingfei Sun \\ Guangdong Academy of Agricultural Sciences, Institute of Veterinary Medicine, \\ 510640 Guangzhou, China
}

\begin{abstract}
Clostridium perfringens is an important pathogen of animals and humans and is the causative agent of Necrotic Enteritis (NE) in poultry. This study focused on the typing of intestinal C. perfringens isolates ( $\mathrm{n}=78,54$ from chickens with NE, 24 from healthy chickens) from China's Southern Guangdong province. All isolates were classified as type A using multiplex PCR assay. The recently described toxin gene associated with NE in chickens, netB was found in $21(26.9 \%)$ of the isolates (18 from chickens with NE and 3 from unrelated chickens with no evidence of $\mathrm{NE}$ ). The in vitro susceptibility results showed that ceftriaxone showed highest activity with lowest $\mathrm{MLC}_{50}$ values $\left(0.25-0.5 \mu \mathrm{g} \mathrm{mL}{ }^{-1}\right)$ followed by cefotaxime $\left(0.25-4 \mu \mathrm{g} \mathrm{mL}{ }^{-1}\right)$, penicilin $\left(0.5-2 \mu \mathrm{g} \mathrm{mL}^{-1}\right)$. However, most of the isolates were resistant to lincomycin, tetracycline, metronidazole and neomycin as evident by the high $\mathrm{MLC}_{50}$. Furthermore, $70.5 \%$ of the isolates $(\mathrm{n}=55)$ were simultaneously resistant to $>4$ antibiotics and $30.8 \%$ of the isolates $(n=24)$ were resistant to all the antimicrobials tested. To the knowledge this is the first study of the prevalence of C. perfringens in broiler chickens in China and the first to examine the isolates for the recently identified novel toxin genes, netB.
\end{abstract}

Key words: China, Clostridium perfringens, molecullar typing, antimicrobial susceptibility, isolates, genes

\section{INTRODUCTION}

Clostridium perfringens (C. perfringens) is the most important clostridial pathogen of poultry, causing a panoply of diseases which include avian malignant disease, gizzard erosions and gangrenous dermatitis (Choi et al., 2003; Piyankarage et al., 1999; Thompson et al., 2006). Necrotic Enteritis (NE) is a common and severe $C$. perfringens-induced disease and considered the most clinically dramatic bacterial enteric disease of poultry (Long et al., 1974). In spite that the disease was first described almost 50 years ago, the virulence factors which lead to the development of $\mathrm{NE}$ have yet to be fully understood (Smyth and Martin, 2010). Pathogenicity of $C$. perfringens is associated with the production of four major toxins including alpha $(\alpha)$, beta $(\beta)$, epsilon ( $\varepsilon$ ) and iota (i). Cp strains are classified into five types (A-E) based on their ability to produce the above toxins (McClane, 2001; Petit et al., 1999). C. perfringens type $\mathrm{A}$ is the most commonly recovered clostridial type from NE cases (Olkowski et al., 2008). Some experimental studies indicate that alpha-toxin which was believed to be the major virulence factor in $\mathrm{NE}$ is not an essential factor in disease development (Keyburn et al., 2006; Van Immerseel et al., 2004).
Recently, Keyburn reported the discovery of a previously unidentified pore forming toxin of $C$. perfringens which they named NetB and the encoding gene was netB. The netB was only identified in strains recovered from chickens with NE. It was neither found in isolates from chickens which did not have NE (Keyburn et al., 2008). Keyburn further reported that netB knockout mutants failed to produce NE in chickens. Results from subsequent studies showed that virulent netB-positive strains may be present in healthy birds at a low incidence and not all $C$. perfringens from chickens with NE were netB-positive (Chalmers et al., 2008; Martin and Smyth, 2009; Nowell et al., 2010), contradictory to the findings of Keyburn (Keyburn et al., 2008).

Recent studies has focused on the role of widespread use of antimicrobials in growth promotion and therapy of infections in food-producing animals as a potential transfer route of antimicrobial-resistant bacteria or the genes encoding antimicrobial resistance into the human food chain (Piddock et al., 2000). The varying, overuse and misuse of the tested antimicrobials in poultry industry has caused serious public health problems in many parts of the world (Khachatourians, 1998).

In China, there are no data on investigation of the new toxin gene $($ netB) and antimicrobial resistance of

Corresponding Author: Mingfei Sun, Guangdong Academy of Agricultural Sciences, Institute of Veterinary Medicine, 510640 Guangzhou, China 
C. perfringens isolated from chickens. This study was designed to determine the occurrence of netB among C. perfringens isolates from chickens with or without $\mathrm{NE}$ and determine its sensitivity to commonly used antimicrobials.

\section{MATERIALS AND METHODS}

Samples: About 103 broiler chickens with history of enteritis on 22 farms and 100 healthy chickens on 10 farms from Guangdong, China were examined. Jejunal swabs of the cecum and small intestine of all birds were collected for bacterial culture.

Bacterial isolation and identification: All swabs were streaked onto $5 \%$ sheep blood agar and incubated overnight at $37^{\circ} \mathrm{C}$ under anaerobic conditions. Isolates were presumptively identified by gram staining and lecithinase production and confirmed biochemically as C. perfringens based on the following tests, lactose and inositol fermentation, stormy fermentation in litmus milk, nitrate reduction, gelatinase production and motility tests (FDA, 1998). Resultant colonies which exhibited a double zone of hemolysis and which failed to grow under aerobic conditions were tentatively identified as C. perfringens. Isolates were stored at $-80^{\circ} \mathrm{C}$ until further testing.

All tentatively identified $C$. perfringens were examined to determine their genotypes by multiplex PCR according to a previously described protocol (Gharaibeh et al., 2010).

Detection of netB: All isolates were examined for the presence of the netB gene as described by Nowell et al. (2010).

Determination of Minimal Inhibitory Concentrations (MICs) of antimicrobials against Clostridium perfringens isolates: $A$ total of 10 antimicrobials shown in Table 1 were used to test their MIC against the isolate confirmed to be $C$. perfringens by PCR. Prior to antimicrobial susceptibility test, isolates were subcultured twice on Brucella Blood Agar plate (BBA). Colonies from

Table 1: Antimicrobials used for MICS testing with their origins

\begin{tabular}{ll}
\hline Antimicrobial & Sources \\
\hline Lincony cin & Sigma \\
Penicillin & Sigma \\
Ceftriaxone & Roche \\
Cefotaxim sodium salt & Sigma \\
Erythromycin & Sigma \\
Tetracycline & Sigma \\
Neomycin sulfate & Amresco \\
Metronidazole & Biolab \\
Tylosin tartrate & Sigma \\
Ofloxacin & Sigma \\
\hline
\end{tabular}

$48 \mathrm{~h}$ BBA were suspended in enriched thioglycollate medium without indicator (Difco), to a turbidity equivalent to that of a $0.5 \mathrm{McF}$ arland standard. An overnight fresh culture of each isolate in Schaedler broth (Difco) that contained approximately $10^{6} \mathrm{CFU} \mathrm{mL}^{-1}$ was used in MIC testing. MIC was performed in duplicates in flat sterile 96 well plates. Final antimicrobial concentration ranged from $0.125-256 \mathrm{mg} \mathrm{mL}^{-1}$. C. perfringens type A (CVCC52, from China Institue of Veterinary Drug Control) was run together with the samples as a reference strain. In each run, both sterility and growth control were included. MIC was read as the lowest antimicrobial concentration that inhibits visible bacterial growth after anaerobic incubation for $18-24 \mathrm{~h}$.

\section{RESULTS AND DISCUSSION}

Clostridium perfringens recovery, genotyping and toxinotyping by PCR: A total of $54 \mathrm{C}$. perfringens isolates were recovered from 103 broiler chickens with history of enteritis and retarded growth on 22 farms and 24 isolates were obtained from 100 healthy broiler chickens on 10 farms. All of the 78 isolates were confirmed as type A by positive PCR reaction for only alpha toxin gene (Fig. 1). Using the netB-specific primers of Nowell et al. (2010), a netB PCR product of the expected $383 \mathrm{bp}$ in length was obtained in 21 out of the 78 isolates $(26.9 \%$ ) (Fig. 2). It was found in normal chickens at a low incidence $(12.5 \%, \mathrm{n}=3)$ while a significantly higher incidence $(33.3 \%, \mathrm{n}=18)$ was found in chickens with NE.

Minimal Inhibitory Concentrations (MICs) of antimicrobials against clostridium perfringens isolates: A total of $78 \mathrm{C}$. perfringens isolates were tested for their susceptibility to 10 antimicrobials and $C$. perfringens CVCC52 was used as a quality control strain in this study.

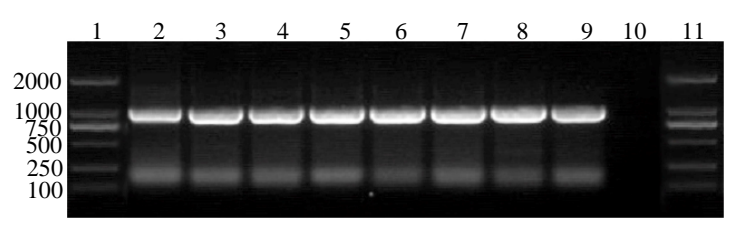

Fig. 1: PCR for alpha gene (900 bp) to confirm isolates to be Clostridium perfringens. Lanes 1 and 11: DNA marker, Lane 2: Clostridium perfringens type A reference strain (CVCC52), Lanes 3-9: Confirmed Clostridium perfringens isolates and Lane 10 : Negative control 


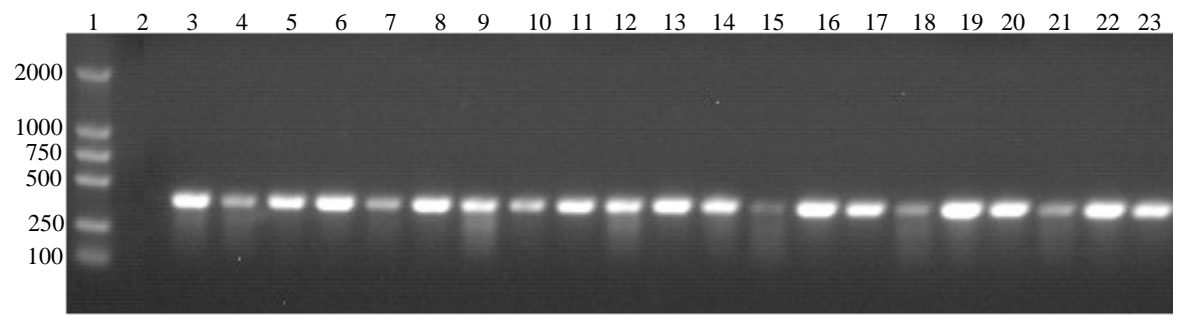

Fig. 2: PCR for netB gene (383 bp) from confirmed Clostridium perfringens type A isolates. Lanes 1: DNA marker, Lane 2: Negative control; Lanes 3-9: netB-positive isolates

Table 2: Frequency distribution of minimal inhibitory concentration of 10 antimicrobials aganist 78 Clostridium perfringens isolates Number of C. perfringens isolates with MIC values $\left(\mu \mathrm{g} \mathrm{mL}^{-1}\right)$

\begin{tabular}{|c|c|c|c|c|c|c|c|c|c|c|c|c|c|c|c|}
\hline \multirow[b]{2}{*}{ Antimicrobial } & \\
\hline & $\leq 0.125$ & 0.25 & 0.5 & 1 & 2 & 4 & 8 & 16 & 32 & 64 & 128 & $\geq 256$ & Range & $\mathrm{MIC}_{50}$ & $\mathrm{MIC}_{90}$ \\
\hline Lincony cin & 0 & 0 & 0 & 0 & 0 & 0 & 0 & 1 & 2 & 0 & 0 & 75 & 16 to $\geq 256$ & $\geq 256.00$ & $\geq 256$ \\
\hline Penicillin & 25 & 15 & 0 & 8 & 11 & 0 & 0 & 0 & 0 & 0 & 0 & 19 & 0.125 to $\geq 256$ & 0.25 & 128 \\
\hline Ceftriaxone & 34 & 16 & 8 & 4 & 2 & 1 & 0 & 0 & 0 & 0 & 0 & 13 & 0.125 to $\geq 256$ & 0.25 & 64 \\
\hline Cefotaxim & 29 & 21 & 4 & 5 & 7 & 2 & 0 & 0 & 0 & 0 & 0 & 10 & 0.125 to $\geq 256$ & 0.50 & 128 \\
\hline Erythromy cin & 0 & 0 & 0 & 0 & 0 & 0 & 0 & 8 & 8 & 12 & 21 & 29 & 16 to $\geq 256$ & 128.00 & $\geq 256$ \\
\hline Tetracy cline & 0 & 2 & 0 & 2 & 7 & 11 & 0 & 0 & 0 & 1 & 0 & 55 & 0.25 to $\geq 256$ & $\geq 256.00$ & $\geq 256$ \\
\hline Neomycin & 0 & 0 & 0 & 0 & 3 & 0 & 7 & 0 & 0 & 4 & 14 & 48 & 2 to $\geq 256$ & 128.00 & $\geq 256$ \\
\hline Metronidazole & 0 & 0 & 0 & 2 & 0 & 5 & 0 & 0 & 0 & 0 & 0 & 71 & 1 to $\geq 256$ & $\geq 256.00$ & $\geq 256$ \\
\hline Tylosin & 0 & 0 & 0 & 1 & 2 & 0 & 0 & 37 & 24 & 1 & 0 & 13 & 1 to $\geq 256$ & 64.00 & $\geq 256$ \\
\hline Ofloxacin & 0 & 0 & 1 & 0 & 0 & 0 & 29 & 32 & 1 & 1 & 0 & 14 & 0.5 to $\geq 256$ & 32.00 & $\geq 256$ \\
\hline
\end{tabular}

Table 2 shows the MIC values and number of resistance of C. perfringens isolated from chickens with NE. C. perfringens isolates showed various resistant to antimicrobials tested in the present study. Most of the isolates showed resistant to lincomycin, tetracycline, metronidazole and neomycin as evident by the high $\mathrm{MIC}_{50}$. Ceftriaxone showed highest activity with lowest $\mathrm{MIC}_{50}$ values $\left(0.25-0.5 \mu \mathrm{g} \mathrm{mL}^{-1}\right)$ followed by cefotaxime $\left(0.25-4 \mu \mathrm{g} \mathrm{mL}^{-1}\right)$ and penicilin $\left(0.5-2 \mu \mathrm{g} \mathrm{mL}^{-1}\right)$. $\mathrm{MC}_{50}$ was relatively high for ofloxacin $\left(8-16 \mu \mathrm{g} \mathrm{mL} \mathrm{m}^{-1}\right)$, tylosin tartrate, $\left(16-32 \mu \mathrm{g} \mathrm{mL}^{-1}\right)$ and erythromycin $\left(16-64 \mu \mathrm{gL}^{-1}\right)$ and high for tetracycline $\left(64-128 \mu \mathrm{g} \mathrm{mL}^{-1}\right)$, neomycin sulfate $\left(64-256 \mu \mathrm{g} \mathrm{mL}^{-1}\right)$, metronidazole $\left(\geq 256 \mu \mathrm{g} \mathrm{m}^{-1}\right)$ and lincomycin $\left(\geq 256 \mu \mathrm{g} \mathrm{mL} \mathrm{L}^{-1}\right)$. $\mathrm{MC}_{90}$ was the lowest for ceftriaxone $\left(64 \mu \mathrm{g} \mathrm{mL}^{-1}\right)$, penicillin $\left(128 \mu \mathrm{g} \mathrm{mL} L^{-1}\right)$ and cefotaxim sodium salt $\left(128 \mu \mathrm{g} \mathrm{mL} \mathrm{m}^{-1}\right)$. All of the other antimicrobials tested had an $\mathrm{MIC}_{90} \geq 256 \mu \mathrm{g} \mathrm{mL}^{-1}$. About $70.5 \%$ of the isolates $(\mathrm{n}=55)$ were simultaneously resistant to $>4$ antibiotics and $30.8 \%$ of the isolates $(n=24)$ were resistant to all of the antimicrobials tested. Ceftriaxone had the highest activity with overall $\mathrm{MIC}_{50}$ and $\mathrm{MIC}_{90}$ of 0.25 and $64 \mu \mathrm{g} \mathrm{mL}{ }^{-1}$, respectively. $\mathrm{MIC}_{50}$ and $\mathrm{MIC}_{90}$ for each antimicrobial are shown in Table 2.

$\mathrm{NE}$ is primarily caused by $C$. perfringens type $\mathrm{A}$ and to a lesser extent type C strains (Thompson et al., 2006). It is worthwhile to note that the C. perfringens type A was the primary cause of the CPE-mediated food poisoning in the industrialized countries and poses significant public health concerns (Fisher et al., 2005). In the present study, typing of $C$. perfringens isolates by multiplex PCR revealed that all isolates were only positive to alpha toxin gene of the four major lethal toxin genes indicating that all C. perfringens isolates represented type A. In Korea, only C. perfringens type A was present in poultry (Yoo et al., 1997). More recently and similar to the present study, only C. perfringens type A from poultry was reported in Finland (Heikinheimo and Korkeala, 2005) and Sweden (Engstrom et al., 2003).

It has recently been reported by Keyburn that netB, a newly discovered pore forming toxin is critical to the development of NE in chickens (Keyburn et al., 2008). They also reported that the isolated netB-positive strains only came from chickens with NE (Keyburn et al., 2008). The definitive discovery of the toxin which enables C. perfringens to produce $\mathrm{NE}$ obviously would represent a major breakthrough in the understanding of the pathogenesis of this serious and economically important disease. Therefore, results from subsequent studies showed that virulent netB-positive strains may be present in healthy birds at a low incidence and not all C. perfringens from chickens with NE were netBpositive (Chalmers et al., 2008; Martin and Smyth, 2009; Nowell et al., 2010). The present investigation revealed a prevalence of netB positive $C$. perfringens isolates of 12.5 and $33.3 \%$ in diseased and healthy broilers, respectively.

This result differs slightly from the investigations performed in Australia, Canada, the United States and Denmark (Chalmers et al., 2008; Keyburn et al., 2008; 
Martin and Smyth, 2009; Nowell et al., 2010) where the prevalence of the net $B$ gene in $C$. perfringens isolates obtained from broilers suffering from NE were generally higher (52-100\%). The US investigations (Martin and Smyth, 2009) also revealed netB-positive C. perfringens in healthy broilers with low prevalence $(8.8 \%$ ) (Martin and Smyth, 2009). The Canadian and Danish investigations revealed higher prevalence (35 and $61 \%$, respectively) than that of the present study (Chalmers et al., 2008; Nowell et al., 2010). It is worth noting that a study reported that the incidence of the netB gene in $C$. perfringens isolates obtained from healthy broilers was higher than that in isolates from chickens suffering from NE (Abildgaard et al., 2010). This finding is not consistent with the hypothesis that netB is the cause of $\mathrm{NE}$ (Keyburn et al., 2008) suggesting that either netB is not essential to the development of $\mathrm{NE}$ in all cases or that net $\mathrm{B}$ is readily lost by the organism. Experimental studies in a disease model are necessary to investigate the disease producing capabilities of both the netB negative strains recovered from cases of $\mathrm{NE}$ and also the netB positive strains recovered from normal chickens.

Minimal inhibitory concentration results revealed that isolates had various susceptibilities to these antimicrobials. In this study, the three antimicrobials that were highly active against $C$. perfringens had the $\mathrm{MIC}_{50}$ of $0.25 \mu \mathrm{g} \mathrm{mL} \mathrm{m}^{-1}$ for both ceftriaxone and penicilin and $0.5 \mu \mathrm{g} \mathrm{mL}^{-1}$ for cefotaxime. Lincomycin, metronidazole and tetracycline were historically known to be among the antimicrobials of choice to treat $C$. perfringens infections in China, their $\mathrm{MLC}_{50}$ was $\geq 256 \mu \mathrm{g} \mathrm{mL}^{-1}$. Similar results were presented from United States, Jordan and Belgium where $\mathrm{MLC}_{50}$ were very high for these antimicrobials (Gharaibeh et al., 2010; Martel et al., 2004; Yoo et al., 1997). These results indicated marked resistance development. Differences in MICs profiles between antimicrobials may reflect the varying, overuse and misuse of the tested antimicrobials in poultry industry in China. Researchers recommend using ceftriaxone, cefotaxime and penicilin for treatment of $C$. perfringens infection in China. Researchers also recommend stopping the use of the popular feed additives that contains lincomycin, metronidazole and tetracycline to control C. perfringens infections.

This study also revealed a high prevalence of antimicrobial-resistant C. perfringens strains. About $70.5 \%$ of the isolates $(\mathrm{n}=55$ ) were simultaneously resistant to more than four antibiotics and $30.8 \%$ of the isolates $(n=24)$ were resistant to all of the antimicrobials tested. This may reflect the widespread or inappropriate use of antimicrobials in chickens resulting in the emergence of multiple-drug resistant strains. They correlate with the amounts and types of antimicrobials consumed by these populations and changes in resistance can be considered as an early warning system for resistance to be expected in potentially pathogenic bacteria (Van den Bogaard et al., 2000). In food-producing animals, a low prevalence and low degree of antimicrobial resistance in the intestinal flora should be considered a distinguishing quality and safety marker.

\section{CONCLUSION}

To the knowledge this is the first study of the prevalence of $C$. perfringens in broiler chickens in China and the first to examine the isolates for the recently identified novel toxin genes, netB. However, the role of netB in the induction of necrotic enteritis needs to be further investigated by determining the disease producing capability of both netB positive strains recovered from normal chickens and netB negative strains recovered from chickens with NE.

\section{ACKNOWLEDGEMENTS}

This research was supported in part by grants from Natural Science Foundation of Guangdong province (No.: 1045106001006126), Science and Technology Development Project for Agriculture from the Department of Guangdong Science and Technology (No: 2010B020307004) and the President's Fund of Guangdong Academy of Agricultural Sciences (No.: 201014).

\section{REFERENCES}

Abildgaard, L., T.E. Sondergaard, R.M. Engberg, A. Schramm and O. Hojberg, 2010. In vitro production of necrotic enteritis toxin $\mathrm{B}, \mathrm{NetB}$, by netB-positive and netB-negative Clostridium perfringens originating from healthy and diseased broiler chickens. Vet. Microbiol., 144: 231-235.

Chalmers, G., H.L. Bruce, D.B. Hunter, V.R. Parreira and R.R. Kulkarni et al., 2008. Multilocus sequence typing analysis of Clostridium perfringens isolates from necrotic enteritis outbreaks in broiler chicken populations. J. Clin. Microbiol., 46: 3957-3964.

Choi, Y.K., M.S. Kang, H.S. Yoo, D.Y. Lee, H.C. Lee and D.Y. Kim, 2003. Clostridium perfringens type A myonecrosis in horse in Korea. J. Vet. Med. Sci., 65: 1245-1247.

Engstrom, B.E., C. Fermer, A. Lindberg, E. Saarinen, V. Baverud and A. Gunnars-Son, 2003. Molecular typing of isolates of Clostridium perfringens from healthy and diseased poultry. Vet. Microbiol., 94: $225-235$. 
FDA, 1998. Bacteriological Analytical Manual (BAM). 8th Edn., Food and Drug Administration, USA.

Fisher, D.J., K. Miyamoto, B. Harrison, S. Akimoto, M.R. Sarker and B.A. McClane, 2005. Association of beta 2 toxin production with Clostridium perfringens type A human gastrointestinal disease isolates carrying a plasmid enterotoxin gene. Mol. Microbiol., 56: 747-762.

Gharaibeh, S., R.A. Rifai and A. Al-Majali, 2010. Molecular typing and antimicr-obial susceptibility of Clostridium perfringens from broiler chickens. Anaerobe, 16: 586-589.

Heikinheimo, A. and H. Korkeala, 2005. Multiplex PCR assay for toxinotyping Clostridium perfringens isolates obtained from Finnish broiler chickens. Lett. Applied Microbiol., 40: 407-411.

Keyburn, A.L., J.D. Boyce, P. Vaz, T.L. Bannam and M.E. Ford et al., 2008. NetB, a new toxin that is associated with avian necrotic enteritis caused by Clostridium perfringens. PLOS Pathog., 4: e26-e26.

Keyburn, A.L., S.A. Sheedy, M.E. Ford, M.M. Williamson, M.M. Awad, J.I. Rood and R.J. Moore, 2006. Alphatoxin of Clostridium perfringens is not an essential virulence factor in necrotic enteritis in chickens. Infect. Immun., 74: 6496-6500.

Khachatourians, G.G., 1998. Agricultural use of antibiotics and the evolution and transfer of antibiotic-resistant bacteria. Can. Med. Assoc. J., 159: 1128-1136.

Long, J.R., J.R. Pettit and D.A. Barnum, 1974. Necrotic enteritis in broiler chick-ens. II. pathology and proposed pathogenesis. Can. J. Comp. Med., 38: 467-474.

Martel, A., L.A. Devriese, K. Cauwerts, K. De Gussem, A. Decostere and F. Haese-Brouck, 2004. Susceptibility of Clostridium perfringens strains from broiler chickens to antibiotics and anticoccidials. Avian. Pathol., 33: 3-7.

Martin, T.G. and J.A. Smyth, 2009. Prevalence of netB among some clinical isolates of Clostridium perfringens from animals in the United States. Vet. Microbiol., 136: 202-205.

McClane, B.A., 2001. The complex interactions between Clostridium perfrigen-senterotoxin and epithelial tight junctions. Toxicon, 39: 1781-1791.
Nowell, V.J., C. Poppe, V.R. Parreira, Y.F. Jiang, R. ReidSmith and J.F. Prescott, 2010. Clostridium perfringens in retail chicken. Anaerobe, 16: 314-315.

Olkowski, A.A., C. Wojnarowicz, M. Chirino-Trejo, B. Laarveld and G. Sawicki, 2008. Sub-clinical necrotic enteritis in broiler chickens: Novel etiological consideration based on ultra-structural and molecular changes in the intestinal tissue. Res. Vet. Sci., 85: 543-553.

Petit, L., M. Gibert and M.R. Popoff, 1999. Clostridium perfringens: Toxinotype and genotype. Trends Microbiol., 7: 104-110.

Piddock, L.J.V., V. Ricci, K. Stanley and K. Jones, 2000. Activity of antibiotics used in human medicine for Campylobacter jejuni isolated from farm animals and their environment in Lancashire, UK. J. Antimicrob. Chemother., 46: 303-306.

Piyankarage, R.H., T. Tajima, S. Sugii and T. Uemura, 1999. Sandwich enzyme-linked immunosorbent assay by using monoclonal antibody for detection of Clostridium perfringens enterotoxin. J. Vet. Med. Sci., 61: 45-47.

Smyth, J.A. and T.G. Martin, 2010. Disease producing capability of netB positive. Microbiology, 113: 25-34.

Thompson, D.R., V.R. Parreira, R.R. Kulkarni and J.F. Prescott, 2006. Live attenuated vaccine based control of necrotic enteritis of broiler chickens. Vet. Microbiol., 113: 25-34.

Van Immerseel, F., J. De Buck, F. Pasmans, G. Huyghebaert, F. Haesebrouck and R. Ducatelle, 2004. Clostridium perfringens in poultry: An emerging threat for animal and public health. Avian Pathol., 33: $537-549$.

Van den Bogaard, A.E.J.M., N. London and E.E. Stobberingh, 2000. Antimicro-bial resistance in pig faecal samples from The Netherlands (five abattoirs) and Sweden. J. Antimicrob. Chemother., 45: 663-671.

Yoo, H.S., S.U. Lee, K.Y. Park and Y.H. Park, 1997. Molecular typing and epid-emiological survey of prevalence of Clostridium perfringens types by multiplex PCR. J. Clin. Microbiol., 35: 228-232. 